\title{
Analytical \& Experimental Analysis of Lightning Stokes Performance \& Protection by MOV Based Simulation Model: A 33 KV Transmission Line Case Study
}

\author{
Arsal Mehmood, Huzaifa Hassana, Faraz Ahmed Baiga, Suhail Ahmed Shaikha \\ Department of Electrical Engineering, Mehran University of Engineering \& Technology, \\ Jamshoro, Pakistan \\ arsalmehmood0713@gmail.com
}

\begin{abstract}
Researchers are working on techniques to mitigate failure rates as low as possible to avoid potential harm, sustain high power efficiency for this a considerable number of estimation studies were already performed and several designs of methodologies were being suggested. The transmission line performs the role of the arteries which maintain the process of transporting electricity in the transmission line. That is why it is important to maintain and manage the costs of these tracks. Surge arrestor and shield wire application are often techniques chosen for defensive strategy in a very technique. By pushing travelling waves towards the electrical equipment mounted on the transmission line, the effects of lightning stoke on the transmission line may cause severe damage to the electrical equipment. In this review, this research study provides a review-based overview of the mechanism of occurrence of lightning along with its impact on the transmission line and the defence methods used to prevent such effects. A MATLAB / SIMULINK 2020a simulation modeling-based analysis for the incidence of lightning on the $33 \mathrm{kV}$ transmission line system is observed in this regard, and a Metal-Oxide surge arrestorbased lightning fault clearance safety scheme is also suggested and discussed.
\end{abstract}

\section{Keywords}

Lightning, Overvoltage, Overhead, Unscheduled, Metal-Oxide Varistor, Surge Arrestor.

\section{Introduction}

According to National Aeronautics and Space Administration (NASA), there are more than 40,000 lightning strokes per day in which less than 100 strokes take place in a second. Lightning is one of the main causes of over-voltage phenomena (Subedi \& Lehtonen, 2019). This results in an unscheduled supply disruption in the power grid when lightning strikes an overhead transmission line. Lightning has the potential to inflict significant harm to the power grid. It allows the travel waves to impact the most vulnerable and costly transmission system equipment connected to both sides. According to a recent research, which says that the a single lightning stoke of about 1 second on an overhead transmission line has an ability to rise the voltage up to $2000 \mathrm{KV}$ (Krider, 2006).

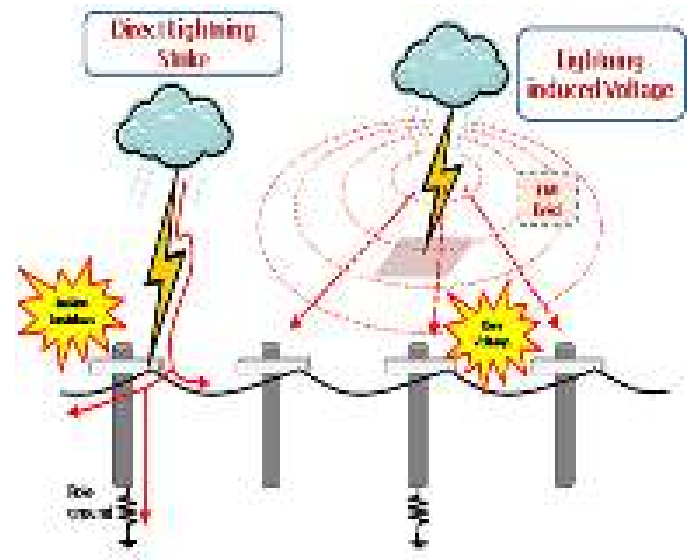

Figure 1: Lightning strike on transmission line. 
It is therefore necessary to maintain stability and reliability in the operation of a power system. Overvoltage is the most common problem that exists in the transmission line and has been introduced in order to solve this problem process and techniques.

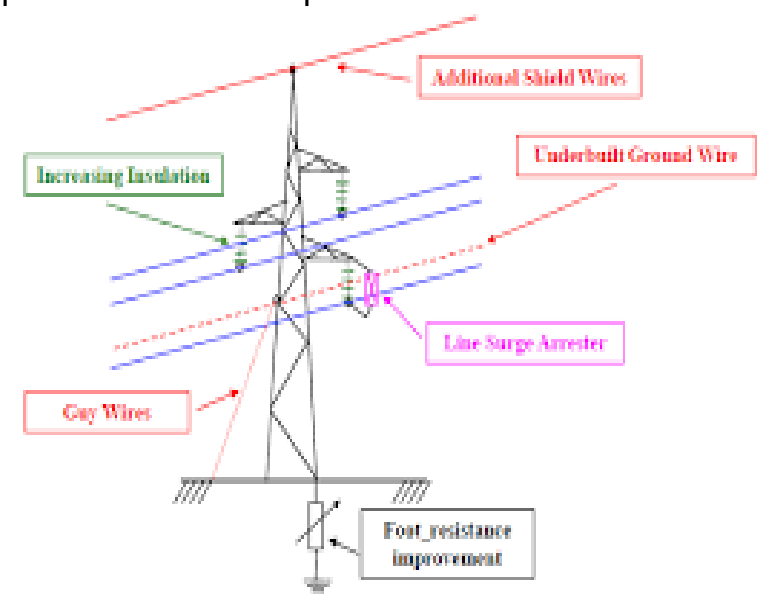

Figure 2: Different method of over voltage protection.

There are two forms of overvoltage, i.e. switching and lightning. Several experiments have been carried out over the last decade and a variety of approaches have been proposed to enhance the efficiency of the power system (Botea, Marinescu, Drăgoi, \& Andrei, 2019). The most critical precaution in the electrical power system is to ensure that the transmission line is shielded from lighting stokes. The accuracy of the lightning performance assessment of the transmission line is very important to make it highly efficient. Metal-oxide-varistor (MOV) and shield wire are the most reliable system used to protect the transmission line from lightning a few days ago (Agrawal \& Nigam, 2014).

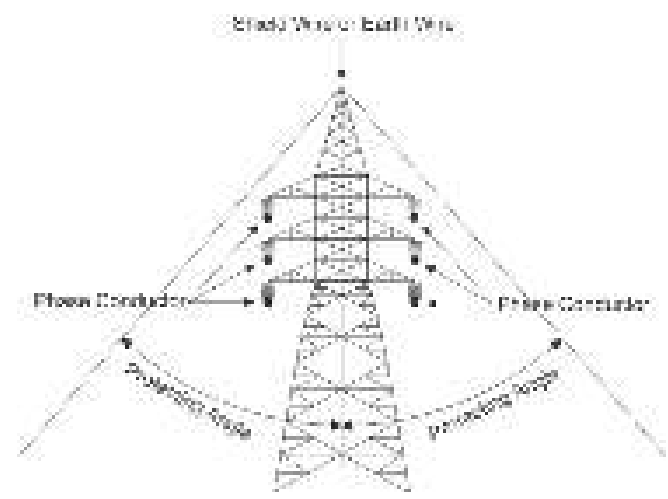

Figure 3: Surge wire protection scheme of transmission line.

Lightning phenomena cause overvoltage, which reduces the reliability of the electrical network and consequently results in an increase in the cost of repairing the transmission line. To avoid lightning strikes, position the ground wire above the top of the tower to reduce annual failure. ( $R, M$, Ayesha, \& Professor, 2007). Metal-oxide-varistor (MOV) is the main source of system protection against lightning and switching. For the defence of air terminals, line lightning rods are used which were invented by the Franklins around 200 years ago (Sharma \& Mahajan, n.d.). Due to the uncertainty of lightning phenomena, the lightning rod cannot always work perfectly due to unexpected shielding failures. Overvoltage in the transmission line may be caused by lightning flashes on or near the transmission line that type of overvoltage is called overvoltage lightning or overvoltage lightning.

\section{Lightning Problem For Transmission Line}

On propagation, the cloud-bottom negative charges generate opposite polarity charges, and this process takes place in the gap capacitance line and the earth and the cloud and the line before the lightning strikes cause the cloud to discharge. There are several forms of potential discharge paths that cause line surge (Morales, Guidi, \& Keune, 2015). 


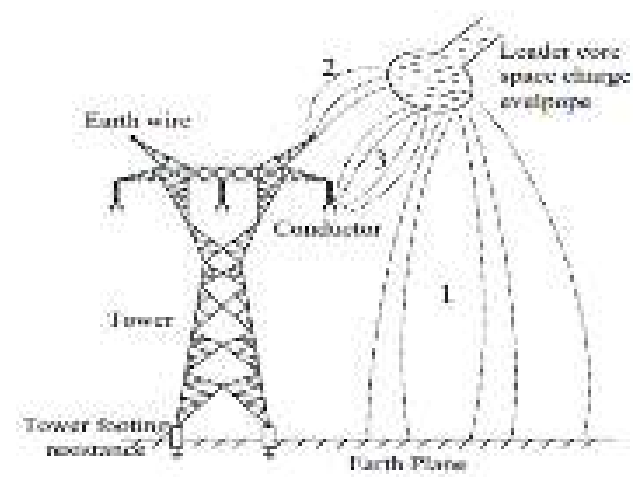

Figure 4: Mechanism of lightning on the transmission line.

On propagation, the cloud-bottom negative charges generate opposite polarity charges, and this process takes place in the gap capacitance line and the earth and the cloud and the line before the lightning strikes cause the cloud to discharge. There are many types of possible discharge paths that cause line surges ( $R$ et al., 2007). It is not a key variable in lightning efficiency. The second unloading direction (2) is between the head of the lightning and the conductor of the earth. This is the direction of discharging potential of these two mediums. The consequential travelling wave comes down the tower and works through the effective impedance of the tower and increases the tower's potential to a certain amount at which the voltage difference between the insulation is sufficient to cause the tower to flash from the back of the tower to the conductor. This was called back flashover mode. Between the phase conductor and the leader core is the 3rd discharging path (3) mode. This makes the capacitance discharge between them and the main discharge current inserted into the phase conductor, so it is important to develop a voltage impedance surge through the insulator string (Agrawal \& Nigam, 2014). At low current, the insulation strength is surpassed, and the discharge path to the earth through the tower is completed. This causes a shielding failure or a direct stroke on the phase conductor. This is called a direct stroke to the phase conductor or a shielding failure. The use of lighting wire or earthing devices is the oldest method of investigation of structure and equipment. In this review, this research study presents a review-based analysis of the mechanism of occurrence of lightning along with its effects on the transmission line and the protection methods used to prevent such effects (Soares, Schroeder, \& Visacro, 2005).

In this regard, a simulation modeling based on the incidence of lightning on the $33 \mathrm{kV}$ transmission line system is observed and the Metal-Oxide surge arrestor-based line stoke safety scheme is also proposed and addressed.

\section{Proposed Model Of $33 \mathrm{Kv}$ Transmission Line}
a. Methodology of analysis

The table below displays the analysis study block diagram.

Table 1: Research Study Block Diagram

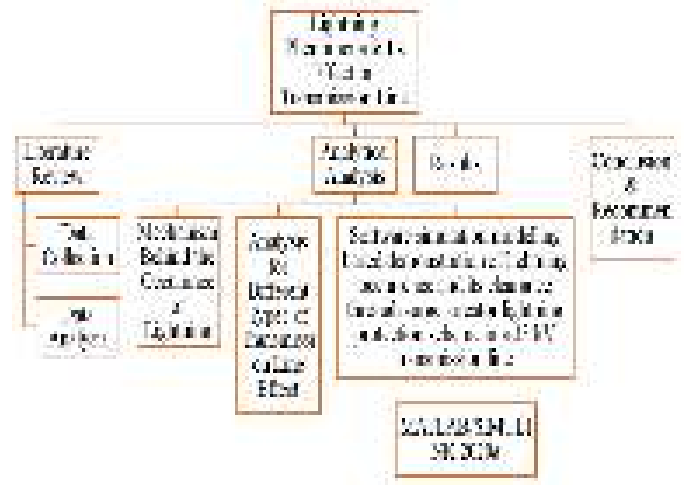




\section{b. Mathematical Modeling}

In our proposed model, we have built a $33 \mathrm{kV}$ equivalent transmission line to feed a load through a $200 \mathrm{~km}$ transmission line. Transmission line consisting of three parts transmitting the end, middle section and receiving end of the system. First, a very high value lightning fault is inserted in the transmission line, which causes the travelling wave pulses, and then different effects are observed. Observation of lightning faults at the different location of the transmission line to investigate the behaviour of the metal-oxide-varistor (MOV) in the grid. Second, the switching fault is found near the load terminal. Only one step of the transmission line is model for simplification. Both parameters that belong to the positive series. The considered 3 phase short-circuit stage of the transmission line is 15000 MVA (Chowdhuri, 2001).

The transmission line is secured by the Metal Oxide Varistor (MOV) which is a voltage suppression system that clamps the transient in the line. The MOV1 consists of 30 columns, while the MOV2 consists of 50 columns. First conduct a simulation modeling on the $33 \mathrm{kV}$ transmission line as shown in the figure below, a proposed model consisting of three ranges in which the first two display the lightning activity on the $33 \mathrm{kV}$ transmission line before the fault is cleared. While the three of them are for after clearing the fault. There are two Metal Oxide Varistor (MOV) at the end of the line, one at the end of the line and the other at the end of the line. Software modeling is carried out using MATLAB / SIMULINK 2020a. In this modeling, there is a demonstration of the lightening surge of around $20 \mathrm{KV}$ exhibits. The length of the $33 \mathrm{KV}$ transmission line is about $16.093 \mathrm{~km}$. The rating of transformer is 5 MVA which is connected to its sending end.

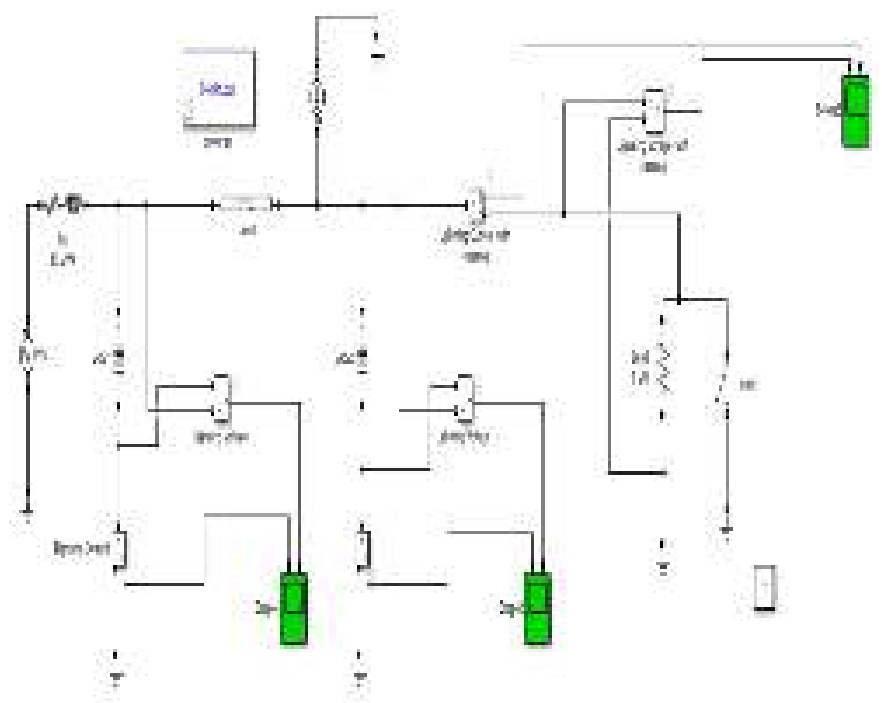

Figure 5: Proposed $33 \mathrm{KV}$ transmission line with MOV protection scheme simulation model

\section{Results}

a) Observation of change in current and voltage at MOV 1 before clearing of Lightning fault

From the below waveform of scope 1 it is observed that at the beginning there is a regular operation of the device for some time as observed by the voltage and current waveform of scope 1 when MOV1 and MOV2 are turned off. Although there is an abnormality in the waveform for the remainder of the period when the fault occurs. 


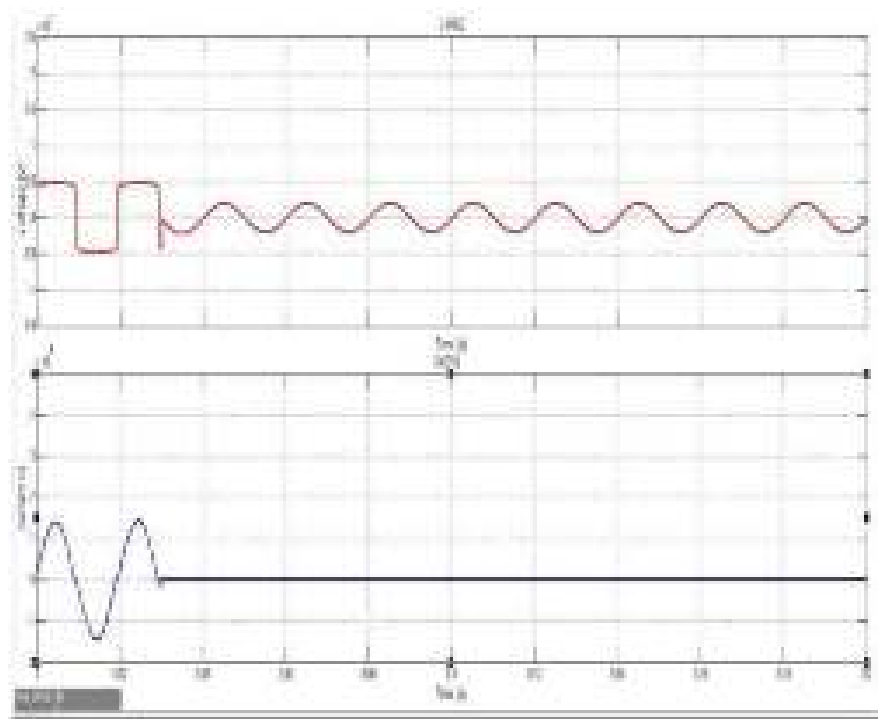

Figure 6: Waveform results for the voltage and current shown by scope 1 when MOV 1 and MOV2 is turn-off.

b) Observation of change in current and voltage at MOV 2 before clearing of Lightning fault

From the below waveform of scope 2 it is observed that at the beginning there is a regular operation of the device for some time as observed by the voltage and current waveform of scope 2 when MOV1 and MOV2 are turned off. Although there is an abnormality in the waveform for the remainder of the period when the fault occurs.

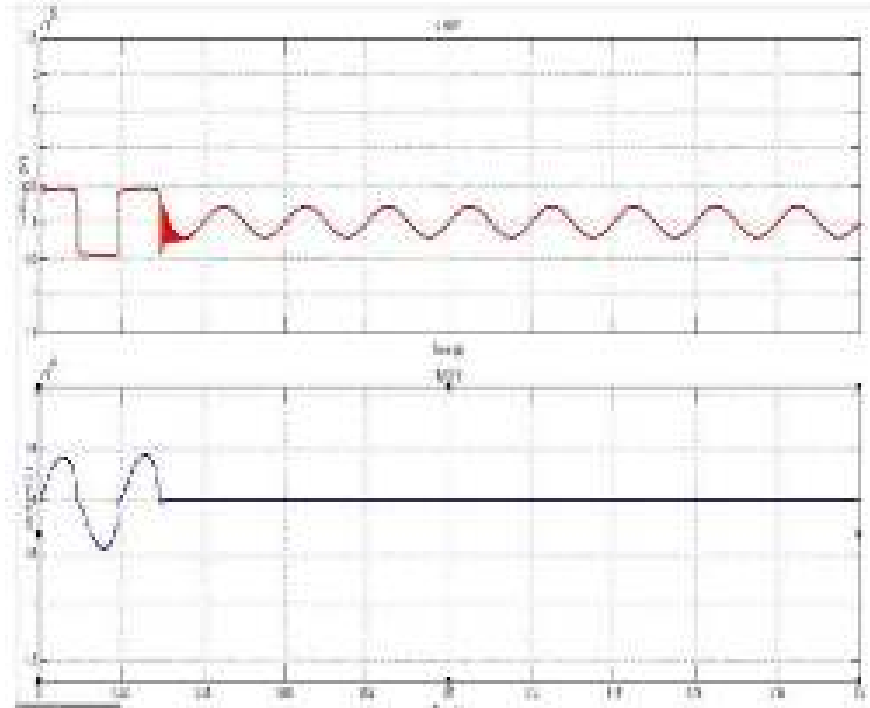

Figure 7: Waveform results for the voltage and current shown by scope 1 when MOV 1 and MOV2 is turn-off.

c) Observation of change in current and voltage after clearing of Lightning fault at $8 \mathrm{MW}$ load

From the waveform below, it is found that there is an abnormality in the system at the beginning for a few cycles. But when the MOV 1 and MOV 2 are turned on, the regular activity of the device will be detected by the voltage and current waveform of the scope 3 . 


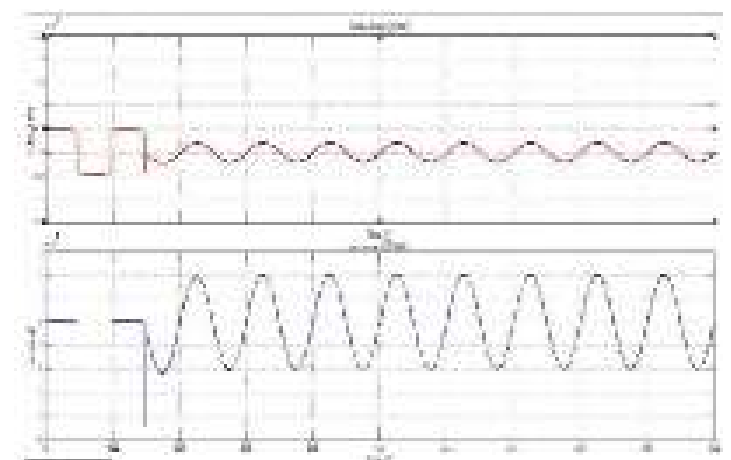

Figure 8: Voltage \& Current waveform observe by the scope 3 when the MOV1 \& MOV2 turn-on.

\section{Conclusion}

Lightning is an unpredictable, capricious, and unpredictable event that can possibly cause severe damage to the transmission line once it occurs. Lightning enter the power system in the form travelling waves towards the components of the line, tower, substation and generating station. Overhead earth wire or shield wires are most commonly use to protect the tower and the line. In this review, the research study intended to analyze the lightning effect on the $33 \mathrm{kV}$ AC transmission line by using MATLAB/SIMULINK 2020a Software.

\section{REFERENCES}

Agrawal, S., \& Nigam, M. (2014). Lightning phenomena and its effect on transmission line. Undefined.

Botea, B., Marinescu, I., Drăgoi, C., \& Andrei, H. (2019). Modeling, Simulation and Analysis of Disturbances and Defects in Low Voltage Instalations. The Scientific Bulletin of Electrical Engineering Faculty, 19(1), 49-57. https://doi.org/10.1515/sbeef-2019-0010

Chowdhuri, P. (2001). Parameters of lightning strokes and their effects on power systems. Proceedings of the IEEE Power Engineering Society Transmission and Distribution Conference, 2, 1047-1051. https://doi.org/10.1109/tdc.2001.971392

Krider, E. P. (2006). Benjamin Franklin and lightning rods. Physics Today, 59(1), 42-48. https://doi.org/10.1063/1.2180176

Morales, J. A., Guidi, G. D., \& Keune, B. M. (2015). Dynamic simulation of lightning strikes on transmission lines based on ATP-matlab. International Journal of Applied Engineering Research, 34390-34395. Retrieved from https://pure.ups.edu.ec/en/publications/dynamic-simulation-oflightning-strikes-on-transmission-lines-bas-6

R, C. S., M, R. P., Ayesha, N., \& Professor, A. (2007). Analysis of the Use of Surge Arresters in Transmission System Using MATLAB/Simulink. International Journal of Advanced Research in Electrical, Electronics and Instrumentation Engineering (An ISO, 3297. https://doi.org/10.15662/IJAREEIE.2016.0503037

Sharma, G., \& Mahajan, A. (n.d.). Simulation of Compensated Transmission Line Protection from Lightning by using Matlab. Retrieved from www.ijert.org

Soares, A., Schroeder, M. A. O., \& Visacro, S. (2005). Transient voltages in transmission lines caused by direct lightning strikes. IEEE Transactions on Power Delivery, $20(2$ II), 1447-1452. https://doi.org/10.1109/TPWRD.2004.839214

Subedi, D., \& Lehtonen, M. (2019). Lightning overvoltages in electrical power system of a power plant. Proceedings of the 2019 20th International Scientific Conference on Electric Power Engineering, EPE 2019. https://doi.org/10.1109/EPE.2019.8777933 